\title{
EL CONFLICTO DE PAREJA COMO VARIABLE ASOCIADA A LA VIO- LENCIA DE GÉNERO CONTRA LA MUJER: CONSECUENCIAS SOBRE LA SALUD SEXUAL Y MENTAL
}

\author{
FELIPE HURTADO ${ }^{1}$, CELIA CISCAR ${ }^{2}$ y MERCEDES RUBIO ${ }^{3}$ \\ ${ }^{1}$ Centro de Orientación Familiar y Sexualidad, Centro de Salud Fuente San Luis, Valencia \\ ${ }^{2}$ Centro de Orientación Familiar y Sexualidad, Hospital Doctor Peset, Valencia \\ ${ }^{3}$ Centro de Orientación Familiar y Sexualidad, Centro de Salud Serrería, Valencia
}

(Aceptado en noviembre de 2003)

La persistencia de un conflicto crónico en la relación de pareja incide directamente en la posibilidad de que se produzcan conductas de violencia de género, así como en la aparición de consecuencias negativas sobre la salud sexual y mental de los miembros de la pareja. En este estudio hemos analizado, con una muestra de 39 parejas heterosexuales que presentaban relaciones insatisfactorias, la asociación entre variables psicosociales y presencia del conflicto, así como la relación entre dicho conflicto y la manifestación de trastornos sexuales y mentales. Los resultados revelan que las estrategias para solucionar conflictos son deficitarias en ambos sexos. Las mujeres manifiestan, a diferencia de los hombres, trastornos en todas las fases de la respuesta sexual. Respecto a la salud mental, todas las personas que manifiestan depresión y la mayoría de las que manifiestan baja autoestima y alto estado de ansiedad, pertenecen al grupo con conflicto de pareja.

Palabras clave: Conflicto de pareja, violencia de género, salud sexual, salud mental, estrategias para solucionar conflictos.

\section{Couple's conflict as associated to gender violence against women: consequences on sexual and mental health}

The persistence of a chronic conflict in couple's relationship is related to an increased probability of gender violence as well to negative consequences on sexual and mental health. In this study we have analyzed the relationship among psychosocial factors and the presence of couple's conflict, and the association between this conflict and the appearance of sexual and mental disorders and/or dysfunctions. For this purpose a sample of 39 heterosexual couples with unsatisfactory couple relationships had been studied. The results revealed that the strategies to cope with conflicts are deficitaires to both sexes. Regarding sexual behavior, women but no men showed dysfunctions in all the phases of the sexual life. As for as the mental health, all the subjects with clinical depressive symptoms, and most of those with low selfesteem and high anxiety-state, were included in the group who reported couple conflict.

Key words: Couple conflict, gender violence, bio-psycho-social factors, sexual health, mental health, strategies to solve conflicts.

Correspondencia: Felipe Hurtado, Centro de Orientación Familiar y Sexualidad, Centro de Salud Fuente San Luis, c/ Arabista Ambrosio Huici, 30, 46013 Valencia, Teléfono: 961972 816, Fax: 963732369. Correo-e: felipe.hurtado@sanidad.m400.gva.es
Agradecimientos: Este estudio se ha financiado con una beca de investigación de la Escuela Valenciana de Estudios en Salud Pública (EVES). N. ${ }^{\odot}$ de Referencia $026 / 2001$. Este estudio ha contado con la ayuda metodológica de la Unidad docente de Medicina Familiar y Comunitaria de Valencia, en concreto con el apoyo de Francisca Gil. 


\section{INTRODUCCIÓN}

Existen actualmente bastantes datos indicadores de que las parejas con relaciones no satisfactorias, cuando se comparan con otras cuya relación es gratificante, muestran una tasa mucho más baja en la interacción de conductas agradables, una tasa más alta de conductas aversivas, o ambas cosas (Bornstein y Bornstein, 1992; Vincent, Weiss y Birchler, 1975). Los estudios sobre la relación entre similitud y satisfacción de pareja indican que los problemas suelen aparecer cuando existen más áreas diferentes dentro de la relación, que áreas de similitud (Shackelford y Buss, 1997). La satisfacción en la pareja surge como resultado de la reciprocidad en contribuir a cada una de las necesidades tanto de los individuos como de la familia.

Los problemas dentro de la relación de pareja son debidos a desajustes que permanecen sin resolver, bien por falta de habilidad en resolver conflictos (Boyle y Vivian, 1996; Cohan y Bradbury, 1997), bien por el uso de estrategias de afrontamiento simples y/o erróneas como la coerción, la violencia física, la respuesta de evitación y el cese de refuerzos para conseguir controlar la conducta y producir los cambios deseados (Shackelford y Buss, 1997). La gran mayoría de expertos en terapia de pareja resaltan la importancia de una comunicación adecuada entre los miembros de la pareja como requisito fundamental para que la misma funcione adecuadamente, lo que además impide el efecto negativo de que se produzcan atribuciones erróneas (Ackerman, 1966; Bornstein y Bornstein, 1992; Bradbury, Beach, Fincham y Nelson, 1996; Fincham, Beach, Harold y Osborne, 1997; Whisman, Dixon y Johnson, 1997).

Otra fuente de problemas dentro de una relación de pareja se relaciona con los cambios que se producen en el ambiente externo y que afectan a su esta- bilidad y equilibrio, como pueden ser la aparición de una tercera persona (Riehl, Frei y Willi, 1994) o el poder reforzador de un trabajo o profesión que puede competir con la relación. La evolución de la sociedad con la incorporación de la mujer al mundo laboral ha alterado la situación de ésta dentro de la pareja tradicional y ha producido cambios en su estructura, creando dificultades en la relación de pareja sobre todo por el reparto no equitativo de las responsabilidades familiares y de las tareas domésticas (Blattberg y Hogan, 1994; Halford y Markman, 1997).

Los rasgos patológicos de personalidad, los trastornos de personalidad y los problemas psicológicos también han recibido atención por parte de los investigadores con el fin de valorar el efecto sobre la estabilidad y satisfacción de pareja (Begin, Sabourin, Lussier y Wright, 1997). Así, al estudiar la relación entre satisfacción de pareja y síntomas depresivos, Fincham, Beach, Harold y Osborne (1997) encuentran que mientras para los hombres el curso causal va desde la depresión a la insatisfacción de pareja, para las mujeres cursa desde la insatisfacción de pareja a la depresión. Este hecho ya fue descrito por Waring (1994) cuando afirmaba que la insatisfacción de pareja era un factor de riesgo tanto para la iniciación como para el mantenimiento de la depresión en la mujer, datos que han sido confirmados en trabajos posteriores tanto para la depresión como para la ansiedad (Assh y Byers, 1996). Respecto a la relación entre personalidad y satisfacción marital, Kosek (1996) realizó un estudio en 107 parejas heterosexuales, encontrando que los rasgos normales de personalidad eran predictores significativos de satisfacción de pareja tanto para hombres como para mujeres.

Los efectos producidos por los problemas de la pareja, ejercen, en las personas afectadas, una gran presión sobre el fun- 
cionamiento psicológico y físico, debido al estrés asociado por los cambios ocasionados en el estilo de vida, siendo también frecuente la investigación que asocia la victimización por violencia, con la presencia de diversas enfermedades y trastornos físicos en la mujer victimizada (Ferrer y Bosch, 2000).

En lo referente a la violencia de género contra la mujer, los medios de comunicación de masas informan continuamente sobre múltiples casos de de este tipo de violencia que además se producen, en la gran mayoría de casos publicados, en el seno de las relaciones de pareja. Los datos disponibles al respecto sugieren que entre un $20 \%$ y un $50 \%$ han sufrido alguna vez actos de violencia en su relación de pareja, y que un $25 \%$ han vivido o viven una situación de violencia (Eriksson, 1997; WHO, 1996, 1998a).

Hay algunos trabajos de publicación reciente que sirven de punto de referencia para nuestro estudio, pues en ellos se analizan distintos factores causales de la violencia en las relaciones de pareja (Arriaga y Oskamp, 1999). Ahora bien, pocos estudios. empíricos sobre la violencia doméstica contra las mujeres han tenido como principal objetivo identificar marcadores de riesgo, dado que hoy por hoy es imposible identificar una característica particular o un conjunto de ellas que puedan ser utilizadas para identificar a futuros individuos agresores o futuras víctimas (Riggs, Caulfield y Street, 2000). Si bien es cierto que, actualmente, se tiene conocimiento de un grupo de factores asociados a la violencia doméstica contra las mujeres y que, por tanto, pueden ser utilizados para identificar a individuos de riesgo (tanto de realizarla como de padecerla), no lo es menos que la literatura científica no aporta datos con suficiente apoyo empírico para muchos de ellos (Caetano, Schafer y Cunradi, 2001). Así, unos estudios hacen referencia a rasgos de perso- nalidad como la impulsividad, la ira, los celos o la dependencia emocional; otros se basan en variables socio-culturales generadoras de estrés, como el bajo nivel cultural, los problemas económicos, el desempleo, la sobrecarga de trabajo, la incorporación de la mujer al mundo laboral, tener muchos hijos o por el contrario la infertilidad; también abundan las investigaciones que resaltan la asociación entre crecer en un hogar con presencia de violencia y/o abusos y la posterior implicación, en la vida adulta, en relaciones de parejas insatisfactorias y con presencia de violencia (Ellis y Malamuth, 2000; Lee, Sun y Chao, 2001).

En cambio, son menos las investigaciones que se centran en variables asociadas a diferencias en la socialización del rol de género, a la existencia de patrones disfuncionales en la comunicación, en la resolución de conflictos y en deficiencias en habilidades sociales que conducen a la cronificación del conflicto en el seno de la relación de pareja y que, a su vez, pueden llevar a la violencia en la relación de pareja (Butler y Wampler, 1999; Milardo, 1998; Widom y HillerSturmhofel, 2001). Interesa por tanto, conocer qué factores ocasionan la violencia en las relaciones de pareja en nuestro medio, con el fin de reducir su frecuencia mediante intervenciones sociales preventivas.

Con el presente estudio nos proponemos los siguientes objetivos específicos:

1. Obtener datos válidos y fiables con relación a factores biopsicosociales precipitantes y mantenedores del conflicto en la relación de pareja, puesto que de su cronicidad depende la aparición de conductas de violencia de género contra la mujer y trastornos sobre la salud mental y / o sexual.

2. Analizar el tipo y grado de desarrollo mostrado en habilidades sociales 
básicas: comunicación, empatía, asertividad y negociación que, como estrategias de afrontamiento eficaces, son necesarias para solucionar conflictos.

3. Cuantificar la frecuencia de trastornos mentales y sexuales, así como de dimensiones o rasgos de personalidad.

\section{MÉTODO}

\section{Sujetos}

Fueron incluidas 39 parejas heterosexuales que solicitaron consulta, bien como consecuencia de tener relaciones de pareja insatisfactorias o bien porque solicitaban tratamiento psico-sexológico por cualquier padecimiento y en las que, tras la evaluación, se diagnosticó la presencia de una relación de pareja insatisfactoria.

Se ha considerado una relación de pareja como insatisfactoria cuando, además de la manifestación de la pareja, se ha confirmado con la anamnesis y con el resultado del cuestionario de ajuste marital (que se describe en el apartado de instrumentos).

El estudio se ha realizado mediante muestreo consecutivo durante un año, en tres Centros de Orientación Familiar y Sexualidad de la ciudad de Valencia, dependientes de la Consellería de Sanidad de la Generalitat Valenciana: Centro de salud Fuente San Luis y Hospital Dr. Peset (área 9 de salud) y Malvarrosa (área 4 de salud).

\section{Instrumentos}

- Escala autoaplicada de ajuste maritalEAM (Locke y Wallace, 1959). Es un instrumento de autoinforme que evalúa la satisfacción marital global. Consta de $\mathbf{1 5}$ items, que evalúan: a) el grado de acuer- do percibido por cada uno de los miembros de la pareja en las diferentes áreas de la vida de pareja (administración familiar, ocio, sexualidad, etc.); b) comportamientos ante la solución de conflictos, y c) el grado de confianza hacia la pareja. La puntuación máxima es de 158 puntos y el punto de corte que diferencia entre parejas armoniosas y conflictivas es 100 . Su fiabilidad es superior a 0,75 en un periodo de cuatro semanas, siendo un instrumento sensible y capaz de reflejar los cambios obtenidos tras el tratamiento.

- Inventario para la Depresión de Beck-BDI (Beck, Rush, Shaw y Emery, 1979). Es un instrumento de autoinforme que consta de 21 items (rango: 0-63 puntos) y mide la intensidad de los sintomas depresivos. El punto de corte más utilizado para discriminar entre la población sana de aquella que manifiesta síntomas depresivos es de 18.

- Cuestionario de Ansiedad EstadoRasgo-STAI-R/E (Spielberger, Gorsuch y Lushene, 1970). (Versión española de TEA, 1982).El inventario de Ansiedad Estado-Rasgo es una prueba de autoinforme que consta de 20 items relacionados con la ansiedad-rasgo y de otros 20 relacionados con la ansiedad-estado. Para la caracterización de los sujetos hemos utilizado los baremos correspondientes a la población española.

- Cuestionario de Personalidad de Eysenck-EPI. (Eysenck y Eysenck, 1964). (Versión española de TEA, 1987). Este cuestionario es una prueba de autoinforme con respuesta SI-NO, que explora dos factores ortogonales y bipolares claramente definidos en la mayoría de los estudios: neuroticismo y extraversión. Además, tiene una escala de Sinceridad con la que se puede identificar a los sujetos que tienden a responder en una dirección "deseable». Tiene dos formas para- 
lelas (A y B) que permiten el retest en un tratamiento experimental, sin la interferencia de los factores de memoria. En esta investigación hemos utilizado la forma B. El punto de corte para la población adulta femenina (baremo profesional) en Neuroticismo es 13, en Extroversión 15 y en Sinceridad está entre 7 y 8 (todos ellos correspondientes al percentil 75). El punto de corte para la población adulta masculina (baremo profesional) en Neuroticismo es de 9, en Extroversión es 16 y en Sinceridad está entre 7 y 8 (correspondientes al percentil 75).

\section{- Escala de Autoestima (EAE)} (Rosenberg, 1965; versión española de Echeburúa y Corral, 1998).). Es un instrumento de autoinforme que consta de 10 items (rango: 10-40 puntos) y que mide el grado de satisfacción que una persona tiene consigo misma. La mitad de los items están planteados de forma afirmativa y la otra mitad de forma negativa, con el objetivo de controlar la aquiescencia. Cuanto mayor es la puntuación, mayor es el grado de autoestima. El punto de corte en la población adulta es de 20 (Ward, 1977).

\section{Recogida y análisis de datos}

Los datos previos a la intervención psicoterapéutica se han recogido en las consultas iniciales de evaluación y diagnóstico. Primero se hicieron consultas individuales con cada miembro de la pareja y después con ambos a la vez. A lo largo del proceso terapéutico se han seguido recogiendo datos referentes a la evolución clínica.

\section{RESULTADOS}

El análisis de datos correspondientes a las variables biográficas pone de mani- fiesto que con respecto a la edad los varones tienen una edad media de $41,6( \pm 8,8)$ años (rango: 29-63 años). Por el contrario, la edad media de las mujeres es ligeramente inferior ( $39 \pm 8$ años), situándose el intervalo de edades entre 23 y 60 años.

En cuanto al estado civil, los hombres en su mayoría $(92,3 \%)$ están casados, el $5,1 \%$ separados y un $2,6 \%$ viudos. La representación porcentual del estado civil para las mujeres de la muestra analizada refleja idéntico resultado que para los hombres en cuanto a la categoría de casadas, pero difiere para el resto, repartiéndose con idénticos porcentajes $(2,6 \%)$ las solteras, divorciadas y separadas.

El nivel cultural indica que los hombres se reparten porcentualmente de mayor a menor de la siguiente forma: en primer lugar los que tienen estudios de nivel medio $(53,8 \%)$, seguidos de los que tienen estudios primarios $(30,8 \%)$ y en tercer lugar los que tienen estudios superiores $(15,4 \%)$. Las mujeres se clasifican en el mismo orden que los hombres según los niveles de estudios, difiriendo ligeramente en los porcentajes: $56,4 \%$ para las de nivel medio, $30,8 \%$ para las de estudios primarios y $12,8 \%$ para las de estudios superiores.

Según categorías profesionales el $84,6 \%$ de los hombres son asalariados frente al $56,4 \%$ de las mujeres. En el caso de las mujeres el $28,2 \%$ son amas de casa; en cambio no hay ni un solo hombre dentro de esta categoría. En el mismo sentido, dentro de la categoría de parados/as solamente hay mujeres $(7,7 \%)$. El $7,7 \%$ de los hombres son profesionales respecto al $5,1 \%$ de las mujeres. Con igual porcentaje $(2,6 \%)$ se reparten sin distinción de sexo la categoría de patrón. Finalmente, en la categoría de pensionista solamente hay $5,1 \%$ de hombres frente a ninguna mujer.

El análisis de datos correspondientes a las variables de relación de pareja pone 
de manifiesto que, con respecto a la satisfacción global de la relación, de los 39 hombres evaluados hay 32 (82\%) que la sienten como conflictiva frente a $7(18 \%)$ que la perciben como armoniosa. En el caso de las mujeres, en cambio, es considerada conflictiva por todas ellas.

Por lo que se refiere a las estrategias para solucionar conflictos, que engloban un conjunto de habilidades sociales básicas para el éxito de la interacción (comunicación, empatía, asertividad y negociación), constatamos que el $59 \%$ de los hombres sienten que acaban cediendo ellos siempre o casi siempre, frente al $38,5 \%$ que dice solucionar los conflictos de pareja de mutuo acuerdo, y al 2,6\% que afirma que son ellas las que acaban cediendo la mayor parte de las veces. En el caso de las mujeres los resultados muestran datos en sentido opuesto: el $59 \%$ de ellas manifiesta que acaban cediendo ante los conflictos, mientras que ellos solamente ceden en el 10\% de los casos según las mujeres, y el $31 \%$ manifiesta llegar al acuerdo mutuo.

La tercera variable se refiere al conjunto de áreas de interacción que conforman la vida de pareja: administración familiar, ocio, afectividad, relaciones con amigos, sexualidad, convencionalismos, filosofía de vida y trato con la familia política. En el ámbito de la sexualidad es donde los hombres manifiestan el mayor porcentaje de desacuerdo $(79,5 \%)$, seguido en segundo lugar por la afectividad (con un $76,9 \%$ ), y en tercer lugar (con unas tasas del $71,8 \%$ ) se encuentran el ocio y la filosofía de vida. Los convencionalismos alcanzan una cota de desacuerdo del $61,5 \%$, siguiendo en orden decreciente las relaciones con amigos (el $59 \%$ ), la administración familiar $(43,6 \%)$ y finalmente los desacuerdos en el trato con la familia política $(35,9 \%)$. Los resultados del grupo de mujeres difieren tanto en el orden de áreas de interacción insatisfactorias, como en los cocientes porcentua- les expresados. La afectividad ocupa el primer lugar de insatisfacción $(92,3 \%)$, seguida por la sexualidad $(84,6 \%)$, los convencionalismos $(74,4 \%)$, la filosofía de vida $(71,8 \%)$, las relaciones con amigos $(66,7 \%)$, el ocio $(64,1 \%)$, las relaciones con la familia política $(59 \%)$ y en último lugar la administración familiar $(46,2 \%)$.

Las variables relativas a la salud sexual muestran los siguientes resultados, diferenciados según la fase de la respuesta sexual, entre ambos sexos. En lo concerniente al deseo sexual para el grupo de hombres, los resultados indican que de los 39 varones, $4(10,3 \%)$ manifiestan deseo sexual hipoactivo con criterios diagnósticos de trastorno. Sin embargo, en el grupo de mujeres lo padecen 22 , esto es, el equivalente al 56,4\% de esta muestra. Asimismo, el trastorno por aversión sexual, que también se encuadra dentro de los trastornos del deseo sexual, es padecido por $5(12,8 \%)$ mujeres frente a ningún hombre. Los datos correspondientes a la fase de excitación sexual indican que muestran trastornos 10 mujeres $(25,6 \%)$ frente a ningún hombre. En cuanto a la fase orgásmica, hay 14 mujeres $(35,9 \%)$ que manifiesta disfunción orgásmica frente a ningún varón. Únicamente hay un hombre que padece eyaculación precoz. Respecto a los trastornos sexuales por dolor, no hay ningún hombre con trastorno en esta fase de la respuesta sexual frente a 2 mujeres $(5,2 \%)$ que padecen dispareunia. Ninguna mujer padece de vaginismo. Finalmente, el estado de satisfacción sexual como medida global de la vivencia de la sexualidad muestra que 11 mujeres $(28,2 \%)$ frente a 4 hombres $(10,2 \%)$ manifiestan insatisfacción sexual.

El conjunto de variables relativas a la salud mental estudiadas muestra los resultados que siguen: En cuanto al estado de ánimo, la gran mayoría de los hombres $(82 \%)$ no presenta alteración alguna, 
frente al $12,8 \%$ con depresión leve y el $5,1 \%$ con depresión moderada. Los resultados para el grupo de mujeres indican que la mayoría $(69,2 \%)$, tienen un estado de ánimo normal, $6(15,4 \%)$ tienen depresión leve, $2(5,1 \%)$ depresión moderada y $4(10,3 \%)$ depresión grave.

Los niveles de autoestima son similares para ambos sexos: 21 hombres $(53,8 \%)$ lo presentan alto y $18(46,2 \%)$ bajo, y en el caso de las mujeres, 19 $(48,7 \%)$ tienen un nivel alto de autoestima, frente a $20(51,3 \%)$ con nivel bajo.

La variable ansiedad se ha medido diferenciado la ansiedad estado como condición emocional transitoria y la ansiedad rasgo como una característica relativamente estable de la persona. Los datos obtenidos sobre los niveles de ansiedad para el grupo de varones refleja que para el estado actual de ansiedad hay $28(71,8 \%)$ que presentan una puntuación percentil por encima de 75 equivalente a un nivel alto, y para el rasgo de ansiedad hay $24(61,5 \%)$ hombres con una puntuación percentil por encima de 75 (rasgo alto de ansiedad). Para el grupo de mujeres, los datos referentes a la ansiedad estado elevada son idénticos a los de los hombres. En cambio, para la ansiedad rasgo existen diferencias, habiendo menor número de mujeres (19; $48,7 \%$ ) con puntuaciones superiores al percentil 75 .

Finalmente, por lo que respecta a los dos factores de personalidad evaluados, los datos muestran que el 51,3\% de hombres (29) son introvertidos frente al $25,6 \%$ (10) que son altamente extrovertidos. Por otro lado, el 71,8\% (28) presentan una puntuación percentil por encima de 75 en neuroticismo, indicativo de inestabilidad emocional, y $9(23,1 \%)$ manifiestan gran estabilidad emocional. En el grupo de mujeres los resultados indican que el $54 \%$ (21) son extrovertidas frente al $33 \%$ (13) que son muy introvertidas. En cuanto a la variable neuroti- cismo, hay un menor número de mujeres respecto al número de varones que presentan una puntuación percentil por encima de $75: 17$ mujeres $(43,6 \%$ de la muestra). El resto (22) obtiene puntuaciones percentiles indicativas de una buena estabilidad emocional.

Una vez han sido descritos los valores porcentuales de las distintas variables estudiadas y comparados los grupos según el sexo de pertenencia, se describen los resultados encontrados mediante el análisis de la intensidad de la asociación entre las distintas variables y el grado de satisfacción en la relación de pareja.

Para medir la intensidad de la asociación hemos utilizado la prueba de Chicuadrado de Pearson dado que tanto la variable dependiente como la independiente son nominales. Los resultados de los análisis de contingencia entre las variables satisfacción de pareja, estrategias para solucionar conflictos y áreas de la vida de la pareja, se muestran en la Tabla 1.

En la variable estrategias para solucionar conflictos los datos evidenciados en el análisis porcentual muestran que tanto el $59 \%$ de los varones como de las mujeres, sentían que eran ellos o ellas las que tenían que ceder como forma de solucionar los conflictos. Globalmente, solo 27 sujetos $(34,6 \%)$ sienten que llegan al acuerdo mutuo frente a $51(65,4 \%)$ que acaban cediendo, hecho que pone en evidencia que carecen en su mayoría de habilidades sociales eficaces para llegar a acuerdos satisfactorios. Los datos del análisis no alcanzan, no obstante, nivel de significación estadística.

Respecto al conjunto de áreas, en todas ellas se pone en evidencia que hay un mayor número de sujetos que sienten la relación de pareja como conflictiva y que además, a excepción de administración familiar y relaciones con familia política, donde el número de sujetos que se sien- 
Tabla 1. Satisfacción de pareja, estrategias para solucionar conflictos, y áreas de la relación de pareja

\begin{tabular}{|c|c|c|c|c|c|}
\hline \multirow[t]{3}{*}{ Variables } & \multicolumn{5}{|c|}{ Satisfacción de pareja } \\
\hline & \multicolumn{2}{|c|}{ Armoniosa } & \multicolumn{2}{|c|}{ Conflictiva } & \multirow{2}{*}{$\chi^{2}$} \\
\hline & $\mathrm{N}$ & $\%$ & $\mathrm{~N}$ & $\%$ & \\
\hline ESTRATEGIAS & & & & & 5,45 \\
\hline Cediendo él & 2 & 2,6 & 25 & 32,1 & \\
\hline Cediendo ella & 0 & $\mathbf{0}$ & 24 & 30,8 & \\
\hline $\begin{array}{l}\text { Mutuo acuerdo } \\
\text { ÁREAS }\end{array}$ & 5 & 6,4 & 22 & 28,2 & \\
\hline Administración & & & & & 2,31 \\
\hline Acuerdo & 4 & 5,1 & 39 & 50,0 & \\
\hline Desacuerdo & 3 & 3,8 & 32 & 41,1 & \\
\hline Ocio & & & & & 7,39 \\
\hline Acuerdo & 5 & 6,4 & 20 & 25,7 & \\
\hline Desacuerdo & 2 & 2,6 & 51 & 65,3 & \\
\hline Afectividad & & & & & 10,81 * \\
\hline Acuerdo & 3 & 3,8 & 9 & 11,6 & \\
\hline Desacuerdo & 4 & 5,1 & 62 & 79,5 & \\
\hline Amigos & & & & & 7,08 \\
\hline Acuerdo & 5 & 6,4 & 24 & 30,8 & \\
\hline Desacuerdo & 2 & 2,6 & 47 & 60,2 & \\
\hline Sexualidad & & & & & $14,75^{*}$ \\
\hline Acuerdo & 3 & 3,8 & 11 & 14,2 & \\
\hline Desacuerdo & 4 & 5,1 & 60 & 77,0 & \\
\hline Convencionalismos & & & & & 5,27 \\
\hline Acuerdo & 4 & 5,1 & 21 & 26,9 & \\
\hline Desacuerdo & 3 & 3,8 & 50 & 64,1 & \\
\hline Filosofía de la vida & & & & & $12,06^{*}$ \\
\hline Acuerdo & 4 & 5,1 & 18 & 23,1 & \\
\hline Desacuerdo & 3 & 3,8 & 53 & 68,0 & \\
\hline Familia política & & & & & 4,46 \\
\hline Acuerdo & 6 & 7,7 & 35 & 44,8 & \\
\hline Desacuerdo & 1 & 1,3 & 36 & 46,1 & \\
\hline
\end{tabular}

Nota: ${ }^{\star} p<0,05$.

ten de acuerdo supera ligeramente a los que se sienten en desacuerdo, en el resto supera el desacuerdo al acuerdo con independencia de sentir la relación como armoniosa o como conflictiva. El análisis de asociación de variables solamente es significativo para afectividad, sexualidad y filosofía de vida.

El análisis de contingencia entre la variable satisfacción de pareja con el conjunto de variables que forman la respuesta sexual (tabla 2), indica que para el conjunto de la muestra la única fase sexual con resultados estadísticamente signifi- cativos es el deseo sexual, dado que el $33,4 \%$ de los sujetos (22 mujeres y 4 hombres), manifiestan deseo sexual alterado y todos señalan tener una relación de pareja insatisfactoria, no habiendo ningún sujeto con alteración del deseo sexual que tenga una relación de pareja armoniosa.

Para el resto de trastornos sexuales, los resultados apuntan en la misma dirección, es decir, todos los sujetos estudiados, ya sean hombres o mujeres, que presentan un trastorno sexual, pertenecen al grupo de los que tienen pareja conflictiva. Sin embargo, seguramente por el escaso número de sujetos estudiados, los resultados no son estadísticamente significativos.

Tabla 2. Satisfacción de pareja y trastornos sexuales

\begin{tabular}{|c|c|c|c|c|c|}
\hline \multirow[t]{3}{*}{ Variables } & \multicolumn{5}{|c|}{ Satisfacción de pareja } \\
\hline & \multicolumn{2}{|c|}{ Armoniosa } & \multicolumn{2}{|c|}{ Conflictiva } & \multirow[b]{2}{*}{$\chi^{2}$} \\
\hline & $\mathrm{N}$ & $\%$ & $\mathrm{~N}$ & $\%$ & \\
\hline Deseo & & & & & $8,55^{\star}$ \\
\hline Trastorno & 0 & 0 & 26 & 33,4 & \\
\hline Normal & 7 & 9,0 & 45 & 57,6 & \\
\hline Aversión & & & & & 1,39 \\
\hline Trastorno & 0 & 0 & 5 & 6,4 & \\
\hline Normal & 7 & 9,0 & 66 & 84,6 & \\
\hline Excitación & & & & & 5,07 \\
\hline Trastorno & 0 & 0 & 10 & 12,8 & \\
\hline Normal & 7 & 9,0 & 61 & 78,2 & \\
\hline Eyaculación precoz & & & & & 1,39 \\
\hline Trastorno & 0 & 0 & 1 & 1,3 & \\
\hline Normal & 7 & 9,0 & 70 & 89,7 & \\
\hline Orgasmo & & & & & 2,57 \\
\hline Trastorno & 0 & 0 & 14 & 18,0 & \\
\hline Normal & 7 & 9,0 & 57 & 73,0 & \\
\hline Dispareunia & & & & & 0,75 \\
\hline Trastorno & 0 & 0 & 2 & 2,6 & \\
\hline Normal & 7 & 9,0 & 69 & 88,4 & \\
\hline Vaginismo & & & & & - \\
\hline Trastorno & 0 & 0 & 0 & 0 & \\
\hline Normal & 7 & 9,0 & 71 & 91,0 & \\
\hline Satisfacción sexual & & & & & 5,33 \\
\hline Trastorno & 0 & 0 & 15 & 19,2 & \\
\hline Normal & 7 & 9,0 & 56 & 71,8 & \\
\hline
\end{tabular}

Nota: ${ }^{\star} p<0,05$. 
El análisis de contingencia entre satisfacción de pareja y las variables relativas a la salud mental (tabla 3), muestra que para el nivel de autoestima la asociación fue significativa, dado que el $41 \%$ de los que tienen una relación conflictiva muestran baja autoestima frente a solo el $7,7 \%$ que presenta baja autoestima dentro del grupo armonioso.

La variable estado de ánimo indica que no hay ningún sujeto que tenga una relación de pareja armoniosa y padezca de depresión. Todos los que padecen depresión, con independencia del sexo y nivel de intensidad, pertenecen al grupo de pareja conflictiva, habiendo mayor número de mujeres afectadas que de hombres $(30,8 \%$ de las mujeres frente al $17,9 \%$ de los hombres). No obstante, si bien los resultados son clínicamente apreciables, no alcanzan niveles de significación estadística.

En cuanto a la ansiedad estado por los mismos motivos argumentados anteriormente, los resultados tampoco alcanzan nivel de significación estadística, aunque clínicamente son apreciables, puesto que dentro del grupo armonioso solamente hay un $6,4 \%$ de sujetos que tienen ansiedad alta; en cambio, dentro del grupo con relación de pareja conflictiva, hay un $65,4 \%$ que muestran nivel alto de ansiedad.

Tabla 3. Satisfacción de pareja y salud mental

\begin{tabular}{llllll}
\hline Variables & \multicolumn{5}{c}{ Satisfacción de pareja } \\
& Armoniosa & Conflictiva \\
& $\mathrm{N}$ & $\%$ & $\mathrm{~N}$ & $\%$ & $\chi^{2}$ \\
\hline Estado de ánimo (BDI) & & & & & 2,47 \\
Depresión ausente & 7 & 9,0 & 52 & 66,6 & \\
Depresión leve & 0 & 0 & 11 & 14,1 & \\
Depresión moderada & 0 & 0 & 4 & 5,1 & \\
Depresión grave & 0 & 0 & 4 & 5,1 \\
Autoestima & & $4,21 *$ & & \\
Baja & 6 & 7,7 & 32 & 41,0 \\
Alta & 1 & 1,3 & 39 & 50,0 \\
Ansiedad Estado & & 3,92 & & \\
Ausencia/mínima & 2 & 2,5 & 11 & 14,1 \\
Moderada & 0 & 0 & 9 & 11,5 \\
Alta & 5 & 6,4 & 51 & 65,4 \\
\hline
\end{tabular}

El último análisis de contingencia realizado sobre la variable satisfacción de pareja con los factores de personalidad se muestra en la Tabla 4.

Tabla 4. Satisfacción de pareja y variables de personalidad

\begin{tabular}{lccccc}
\hline Variables & \multicolumn{5}{c}{ Satisfacción de pareja } \\
& $\mathrm{N}$ & $\%$ & $\mathrm{~N}$ & $\%$ & $\chi^{2}$ \\
\hline Ansiedad Rasgo & & & & & 2,71 \\
$\quad$ Ausencia/mínima & 1 & 1,3 & 19 & 24,3 & \\
$\quad$ Moderada & 2 & 2,5 & 13 & 16,7 & \\
$\quad$ Alta & 4 & 5,1 & 39 & 50,0 & \\
Neuroticismo & & & & & 0,86 \\
$\quad$ Ausente/mínimo & 2 & 2,5 & 16 & 20,6 & \\
$\quad$ Moderado & 1 & 1,3 & 14 & 17,9 & \\
$\quad$ Alto & 4 & 5,1 & 41 & 52,6 & \\
Extroversión & & & & & 1,18 \\
$\quad$ Ausente/mínimo & 3 & 3,9 & 30 & 38,4 & \\
$\quad$ Moderado & 2 & 2,5 & 12 & 15,4 & \\
$\quad$ Alto & 2 & 2,5 & 29 & 37,2 & \\
\hline
\end{tabular}

La mitad de la muestra $(55,1 \%)$ manifiesta alta ansiedad rasgo, perteneciendo el $50 \%$ al grupo de pareja conflictiva y solamente el $5,1 \%$ al grupo armonioso. En la variable neuroticismo ocurren datos similares, habiendo un $57,7 \%$ de la muestra total con alto nivel, repartiéndose el $52,6 \%$ en el grupo con conflicto y el $5,1 \%$ en el armonioso. Finalmente, la variable extroversión muestra un número ligeramente mayor de sujetos altamente introvertidos/as $(42,3 \%)$ que de altamente extrovertidos $(39,7 \%)$.

\section{DISCUSIÓN}

En este trabajo se ha estudiado una muestra de parejas heterosexuales que manifestaban un conflicto de relación de pareja, confirmada tras diagnóstico psicosexológico y que demandaban terapia de pareja. Es una muestra que abarca distintos estados civiles, niveles culturales, edades y profesiones, pero dado que acuden a 
centros de asistencia pública, cabe pensar que no estén representadas las parejas con recursos socioeconómicos altos, que pueden utilizar recursos de asistencia privada.

Partimos de la base, argumentada científicamente, de que la presencia de un conflicto crónico de pareja indica la presencia de habilidades sociales insuficientes o inadecuadas, para solucionar los conflictos a los que se tienen que enfrentar las parejas en sus relaciones y que, de la persistencia del conflicto, se derivan consecuencias importantes sobre la salud sexual, afectiva y mental, junto con alta probabilidad de aparición de conductas de violencia de género hacia la mujer, por efecto de las diferencias en la socialización del rol de género presente en culturas masculinas como la nuestra, actuando de esta manera el conflicto crónico de pareja como factor predictor de violencia de género hacia la mujer. Por lo tanto, conociendo primero qué factores intervienen en la cronificación del conflicto de pareja, y actuando después sobre los mismos, podríamos prevenir o minimizar las consecuencias negativas sobre la salud y la aparición de las conductas de violencia entre sexos.

\section{a) Variables biopsicosociales}

Diversos investigadores se han planteado, igual que nosotros, examinar la relación entre diversas variables biopsicosociales y la violencia de género contra las mujeres en el seno de la relación íntima de pareja, que es donde se produce el mayor porcentaje de la misma y que además es uno de los mayores problemas de salud pública en los diferentes países. Como sustento de esta afirmación, Caetano, Schafer y Cunradi (2001) informan que en Estados Unidos éste es el mayor problema de salud pública. En su investigación sobre los resultados obtenidos de un estudio nacional realizado en 1995, el $23 \%$ de parejas negras, el $11,5 \%$ de parejas blancas y el $17 \%$ de hispanas tuvieron incidentes de violencia del hombre contra la mujer en el seno de la relación íntima en los 12 meses precedentes al estudio.

En un estudio realizado sobre una muestra de 54 hombres de diferentes razas y culturas (blancos, negros e hispanos), que habían realizado actos de agresiones tanto verbales como físicas contra sus parejas, Soler, Vinayak y Quadagno (2000) incluyeron además de variables biográficas y sociales, alguna variable biológica como el nivel de testosterona del agresor y alguna variable conductual como el abuso de sustancias adictivas. Encontraron que solamente los niveles elevados de testosterona, el bajo estatus socioeconómico, y el consumo del alcohol, contribuían a explicar la varianza encontrada tanto en agresiones verbales como en físicas.

En esta línea Klevens (2001) estimando la magnitud del problema de la violencia contra la mujer en el contexto de la relación íntima de pareja, identificó diversos factores relacionados con el riesgo de su ocurrencia: bajo nivel cultural, bajo nivel socioeconómico, tener muchos hijos, mantener frecuentes conflictos de pareja durante mucho tiempo, la ausencia de otros miembros de la familia, excepto los hijos, viviendo en el hogar, una historia de abuso en la familia de origen, la existencia de prohibiciones impuestas por el marido respecto a actividades sociales, planificación familiar y sexualidad, trabajo, etc. Los factores más claramente relacionados fueron el frecuente conflicto de la pareja y las prohibiciones impuestas.

Nuestros datos confirman, en las parejas estudiadas, que el conflicto crónico de pareja es una constante para el $91 \%$ de la muestra. No obstante, constatamos diferencias importantes asociadas al género, 
ya que mientras que para el $100 \%$ de las mujeres la relación es conflictiva, no alcanza el mismo nivel para el grupo de hombres $(82 \%)$.

Los datos biográficos y socioculturales no muestran diferencias destacables, ya que se reparten de forma similar entre ambos sexos y, asimismo, se distribuyen de forma equiparable a como lo hacen en la población general de las áreas de salud.

\section{b) Satisfacción de pareja, áreas y habilidades sociales}

El estudio de las variables que afectan a la satisfacción en la relación de pareja, ha ocupado a diversos investigadores ya que de sus resultados se derivan las claves para realizar programas preventivos y tratamientos eficaces.

Uno de los factores clave, que ha sido apuntado en recientes investigaciones, es la habilidad para manejar conflictos, puesto que las parejas satisfechas se diferencian de las parejas insatisfechas no en la ausencia de conflictos, sino en los diferentes estilos que utilizan para manejarlos (Greeff, 2000; Scaturo, Hayes, Sagula y Walter, 2000). En este sentido Collins y Feeney (2000) concluyen de sus investigaciones realizadas sobre estilos de interacción en relaciones conyugales, que las parejas que mejor funcionan son aquellas que se comprometen en estilos interactivos de apoyo y apego, es decir, utilizan la empatía y la reciprocidad. Por su parte, Greeff y Bruyne (2000) realizaron un estudio sobre una muestra de 57 parejas que llevaban casadas como mínimo diez años. El objetivo de su estudio era investigar qué estilo de manejar los conflictos tenía mayor asociación significativa con la satisfacción de pareja. Los resultados mostraron que el estilo colaborativo basado en la búsqueda de soluciones satisfactorias para ambos, era el que presentaba la más alta correlación con satisfacción de pareja. Sin embargo, cuando uno o ambos miembros de la pareja utilizaba un estilo competitivo, es decir, luchar por tener la razón e imponer los criterios, los resultados mostraron la más baja asociación con satisfacción de pareja. Los resultados sobre los diferentes estilos fueron interpretados por estos investigadores, en términos de diferencias culturales y de género.

En una línea similar apuntan los resultados de nuestro trabajo, donde se puede observar que las parejas con relación de pareja conflictiva muestran estrategias inadecuadas para solucionar conflictos, estrategias que como ya se indicó, engloban el conjunto de habilidades sociales básicas para el éxito de la interacción social: comunicación, asertividad, empatía y negociación. Vemos que expresan sentimientos contrarios, puesto que tanto el $59 \%$ de los hombres como en el mismo porcentaje de mujeres, sienten que, ellos o ellas, acaban cediendo siempre o casi siempre ante la resolución de los conflictos de pareja. Solamente sienten que llegan a acuerdos mutuos el $38,5 \%$ de los hombres y el $31 \%$ de las mujeres. Sin embargo, hay diferencias de género en cuanto a la prioridad que se da a las diferentes áreas de interacción y la cota de malestar manifestado. Para las mujeres es la afectividad seguida de la sexualidad, donde se manifiesta el mayor conflicto con su pareja. Mientras que para los hombres el orden de prioridad de las dos áreas más importantes, está invertido y con menor cota de desacuerdo. Estas dos áreas, junto a la filosofía de vida, son las que muestran el mayor peso en la presencia de un conflicto de pareja.

La comunicación resalta como una variable fundamental en el mantenimiento de la satisfacción de pareja y en la prevención de la violencia de género en el seno de la relación íntima marital. Berns, Jacobson y Gottman (1999) han 
comparado los patrones de interacción de 47 parejas donde el marido era violento, 28 parejas con conflicto pero sin que el marido fuera violento y 16 parejas satisfechas. Tanto agresores como agredidas mostraron menos comunicación positiva y más comunicación negativa que los hombres y mujeres de las parejas donde no había violencia. Además los hombres agresores mostraron niveles significativamente más altos de demandas amenazantes y abandonos que los otros hombres no agresivos. Las mujeres agredidas, por su parte, se mostraron más demandantes de cambios que las mujeres de parejas no violentas.

Por su parte, Lawrence y Bradbury (2001) obtuvieron datos de 56 parejas con agresiones físicas y predictores de ajuste de pareja, confirmando que la insatisfacción de pareja era más común entre las parejas donde se daban agresiones que entre las que no se daban tales conductas ( $70 \%$ frente al $38 \%$ ), y también dependiendo de la severidad de la agresión. La agresión permaneció como un predictor estable de insatisfacción una vez controladas otras variables como la presencia de eventos estresantes y la comunicación negativa.

\section{c) Salud sexual}

La presencia de disfunciones sexuales en mujeres con problemas de insatisfacción en la relación de pareja ha sido resaltado en diferentes investigaciones (p.ej., Hayden, 1999). Este autor encuentra que la inconsistencia del orgasmo en la mujer muestra una asociación significativa con insatisfacción de pareja, pero que como es lógico esperar, la asociación más fuerte de la dificultad orgásmica se produce con insatisfacción sexual.

Nuestros resultados apuntan, como era previsible y en concordancia con los resultados de la investigación anterior, que para el conjunto de mujeres hay alteraciones en diferentes proporciones en todas las fases de la respuesta sexual humana, puesto que todas, sin exclusión, sienten su relación como insatisfactoria. En cambio, en el conjunto de hombres, que como ya apuntábamos no todos sentían su relación de pareja de forma conflictiva, solamente hay $4(10 \%)$ que manifiestan trastorno del deseo sexual y por consiguiente, insatisfacción sexual, perteneciendo éstos a los que sienten conflictiva su relación. Por lo tanto, todos los sujetos estudiados que manifiestan trastorno sexual, con independencia del sexo, pertenecen al grupo de los que tienen pareja conflictiva.

Del conjunto de trastornos sexuales, solamente el deseo sexual hipoactivo muestra asociación estadísticamente significativa con la insatisfacción crónica de pareja. Para el resto de trastornos sexuales (manifestados con exclusividad en las mujeres, a excepción de un hombre con eyaculación precoz), si bien los resultados pueden ser clínicamente significativos, no lo son desde el punto de vista estadístico debido probablemente al número de sujetos estudiados.

\section{d) Salud mental}

Las consecuencias que sobre la salud mental tiene el sufrimiento crónico de un conflicto de pareja y, cómo no, de la violencia de género, han sido resaltadas por diferentes investigaciones (p.ej., Kernic, Wolf y Holt, 2000; Lipman, MacMillan y Boyle, 2001; Plitchta y Falik, 2001). Robbins (1999) indica que la depresión es una consecuencia previsible del conflicto de pareja. Por su parte, Blaustein (1999) indica que el conflicto crónico genera tanto en ambos miembros de la pareja como en los hijos, síntomas depresivos y baja autoestima. Woods (2000) expresa que la prevalencia del trastorno 
de estrés postraumático y el riesgo de suicidio es muy alto en las mujeres que han sufrido abusos tanto físicos como psicológicos en el seno de una relación íntima de pareja, correlacionando positivamente con la severidad de la agresión experimentada.

Estimando la frecuencia de diferentes formas de violencia sexual y su asociación con problemas de salud mental, Ramos, Saltijeral, Romero, Caballero y Martínez (2001) encuentran, en una muestra de 345 mujeres que habían sufrido diferentes formas de violencia sexual, que una de cada cinco había experimentado violencia sexual en el contexto de una relación íntima y que existía una asociación significativa con depresión, ideación suicida, intentos de suicidio y uso de drogas psicoactivas. Resultados similares encuentran Coker, Pope, Smith, Sanderson y Hussey (2001) en un estudio realizado sobre 1152 mujeres atendidas en centros clínicos de Estados Unidos entre 1997 y 1998, por haber sufrido palizas o agresiones físicas. El $18 \%$ del total había experimentado la agresión de su propia pareja y mostraba alteraciones en su salud mental: ansiedad, depresión, uso de drogas y bajo apoyo social.

En nuestra serie, confirmando los datos encontrados en las investigaciones reseñadas, los resultados reflejan que para el conjunto de la muestra, con independencia del sexo, todas las personas con trastorno del estado de ánimo, y la mayoría de los que presentan baja autoestima o alto estado de ansiedad, pertenecen al grupo de personas con conflicto crónico de pareja. Centrándonos en las mujeres, por ser el grupo que manifiesta al $100 \%$ la relación como conflictiva, los datos sobre las consecuencias del conflicto crónico para la salud mental indican que hay más mujeres con trastornos del estado de ánimo que hombres (31\% frente a $18 \%$ ), con baja autoestima ( $51 \%$ de mujeres frente al $46 \%$ de hombres) y con niveles moderado-altos de ansiedad ( $87 \%$ de mujeres frente a $79 \%$ de hombres).

No obstante, hay que decir que estos datos sobre las consecuencias del conflicto crónico de pareja sobre la salud mental aunque son clínicamente apreciables y se hallan en la misma línea de resultados obtenidos en otras investigaciones, no son estadísticamente significativos, salvo para el caso de la autoestima.

\section{e) Personalidad}

Las variables relacionadas con la personalidad han sido objeto de estudio por su probable asociación con la violencia de género y la existencia de conflicto crónico en la relación de pareja. Waltz, Babcock, Jacobson y Gottman (2000) proponen una tipología tripartita de hombres agresores de mujeres dentro de la relación de pareja, basada en la severidad de la violencia, la extensión de la violencia, y la presencia de trastornos de la personalidad. Usaron datos sobre 75 hombres que habían realizado actos de violencia contra su pareja y sobre 32 parejas en conflicto, pero donde no habían ocurrido actos de violencia. Encuentran que, en general, los hombres agresores fueron significativamente más violentos tanto dentro como fuera de la relación de pareja y, además, presentaron numerosos síntomas psicológicos, en comparación con los no agresores. Asimismo, se confirmaron diferencias en las familias de origen, en cuanto a la existencia de violencia, el apego y las habilidades de comunicación.

Investigaciones como la de Caetano, Schafer y Cunradi (2001) concluyen que la personalidad impulsiva, junto al consumo del alcohol y a la presencia de conducta violenta, son precursores comunes de la violencia de género del hombre contra la mujer, si bien plantean la dis- 
yuntiva de si, en el caso del alcohol, éste actúa como precursor de violencia (por su efecto fisiológico desinhibidor) o si más bien el varón agresor utiliza conscientemente el consumo de alcohol como excusa para ejercer su violencia.

El rasgo alto de ansiedad y sus aliados (la afectividad negativa y el neuroticismo) se han encontrado frecuentemente asociados con insatisfacción conyugal, pero se han realizado pocos esfuerzos para identificar los procesos a través de los que ejercen sus influencias (Caughlin, Huston y Houts, 2000). Estos últimos autores hicieron un estudio longitudinal de 13 años en parejas recién casadas y en aquellas en que alguno de sus miembros tenía alto rasgo de ansiedad: se evidenció desde el principio un impacto negativo sobre la satisfacción de la relación de pareja, manteniéndose su efecto de manera estable a través del tiempo. Nosotros también encontramos que, en el conjunto de la muestra, el $50 \%$ de las personas con sentimiento de pareja conflictiva presentaba alto rasgo de ansiedad, frente a sólo el $5 \%$ con alta ansiedad rasgo y relación armoniosa de pareja. Según sexos, hay mayor tasa de hombres que de mujeres con alto ansiedad-rasgo, al igual que sucede en la población general.

En cuanto al factor de personalidad neuroticismo-estabilidad emocional, si bien no se alcanzan resultados estadísticamente significativos, los resultados son clínicamente significativos, puesto que el $52,6 \%$ de las personas que sienten su relación de pareja como conflictiva muestran elevada inestabilidad emocional contra solamente el $5,1 \%$ de personas con igual nivel pero con una relación armoniosa de pareja. Respecto a las diferencia de sexo, hay mayor porcentaje de hombres que de mujeres con neuroticismo elevado.

Finalmente la dimensión extroversiónintroversión es la única que no ha mostrado resultados estadística ni clínica- mente significativos respecto a la asociación con la insatisfacción crónica de pareja, puesto que se reparten en similares proporciones, con independencia del sexo, los sujetos introvertidos y los extrovertidos tanto entre los que sienten armoniosidad o conflictividad en su relación. En ninguno de los estudios que hemos revisado, hemos constatado el estudio de esta variable de personalidad por lo que no podemos comparar nuestros resultados. En todo caso, pensamos que es una faceta importante de personalidad, a la que se le debería dedicar un análisis en profundidad con el fin de confirmar o, en su caso, desechar su potencialidad predictiva como rasgo precursor $y / o$ mantenedor del conflicto de pareja y de la violencia de género.

\section{REFERENCIAS BIBLIOGRÁFICAS}

Ackerman, N.W. (1966). Treating the troubled family. Nueva York: Basic.

Arriaga, X.B., y Oskamp, S. (1999). Violence in intimate relationships. Thousand Oaks, CA: Sage Publications.

Assh, S.D., y Byers, E.S. (1996). Understanding the co-occurrence of marital distress and depression in women. Journal of Social and Personal Relationships, 13, 537-552.

Beck, A.T., Rush, A.J., Shaw, B.F., y Emery, G. (1979). Cognitive Therapy of depression. Nueva York: Guilford Press.

Begin, C., Sabourin, S., Lussier, Y., y Wright, J. (1997). L'evaluation subjective directe des emotions ressenties au sein du couple. International Journal of Psychology, 32, 315-327.

Berns, S.B., Jacobson, N.S., y Gottman, J.M. (1999). Demand-withdraw interaction in couples with a violent husband. Journal of Consulting and Clinical Psychology, 67, 666-674.

Blattberg, K.J., y Hogan, J.D. (1994). Marital distress across the mid-life transition among middle-class Caucasian women. Psychological Reports, 75, 497-498. 
Blaustein, K.G. (1999). The effects of interparental conflict and parent-child conflict on young adult adjustment: A model. Dissertation Abstracts International: Section$\mathrm{B}$ : The Sciences and Engineering, 60, 1842.

Bornstein, P.H., y Bornstein, M.T. (1992). Terapia de pareja. Enfoque conductual-sistémico. Madrid: Pirámide.

Boyle, D.J., y Vivian, D. (1996). Generalized versus spouse specific anger/hostility and men's violence against intimates. Violence and Victims, 11, 293-317.

Bradbury, T.N., Beach, S.R., Fincham, F.D. y Nelson, G.M. (1996). Attributions and behavior in functional and dysfunctional marriages. Journal of Consulting and Clinical Psychology, 64, 569-576.

Butler, M.H., y Wampler, K.S. (1999). A metaanalytical update of research on the couple communication program. American Journal of Family Therapy, 27, 223-237.

Caetano, R., Schafer, J., y Cunradi, C.B. (2001). Alcohol-related intimate partner violence among white, black, and Hispanic couples in the United States. Alcohol Research and Health, 25, 58-65.

Caughlin, J.P., Huston, T.L., y Houts, R.M. (2000). How does personality matter in marriage? An examination of trait anxiety, interpersonal negativity, and marital satisfaction. Journal of Personality and Social Psychology, 78, 326-336.

Cohan, C.L., Bradbury, T.N. (1997). Negative life events, marital interaction, and the longitudinal course of newlywed marriage. Journal of Personality and Social Psychology, 73, 114-128.

Coker, A.L., Pope, B.O., Smith, P.H., Sanderson, M., y Hussey, J.R. (2001). Assessment of clinical partner violence screening tools. Journal of American Medical Women Association, 56, 19-23.

Collins, N.L., y Feeney, B.C. (2000). A safe haven: an attachment theory perspective on support seeking and caregiving in intimate relationships. Journal of Personality and Social Psychology, 78, 1053-1073.

Echeburúa, E., Corral, P. (1998). Manual de violencia familiar. Madrid: Siglo XXI.

Ellis, B.J., y Malamuth, N.M. (2000). Love and anger in romantic relationships: a discrete systems model. Journal of Personality, 68, 525-556.

Eriksson, M. (1997). (Ponente) Violencia contra las mujeres (A4.0250/97), [documento WWW] http:// nodo 50.ix.apc.org/muje$\mathrm{resred} /$ violencia - ue/htm.

Eysenck, H.J, y Eysenck, S.B.G. (1964). Eysenck Personality Inventory. Londres: Hodder and Sotughton, Educational. (Versión española, TEA ediciones, 1987).

Ferrer, V.A., y Bosch, E. (2000). Algunas consideraciones sobre las consecuencias de la violencia doméstica sobre la salud de la víctima. Cuadernos de Medicina Psicosomática y Psiquiatría de Enlace, 55/56, 37 . 45.

Fincham, F.D., Beach, S.R.H., Harold, G.T., y Osborne, L.N. (1997). Marital satisfaction and depression: Different causal relationships for men and women? Psychological Science, 8, 351-357.

Greeff, A.P. (2000). Characteristics of families that function well. Journal of Family Issues, 21, 948-962.

Greeff, A.P., y de Bruyne, T. (2000). Conflict management style and marital satisfaction. Journal of Sexual and Marital Therapy, 26, 321-334.

Halford, W.K., y Markman, H.J. (Eds.) (1997). Clinical handbook of marriage and couples interventions. Chichester: John Wiley \& Sons.

Hayden, R.L. (1999). Women's orgasm inconsistency in heterosexual marriage. (sexual dysfunction, human sexuality). Dissertation Abstracts International: Section-B: The Sciences and Engineering, 60 (5-B), 2342.

Kernic, M.A., Wolf, M.E., y Holt, V.L. (2000). Rates and relative risk of hospital admission among women in violent intimate partner relationships. American Journal of Public Health, 90, 1416-1420.

Klevens, J. (2001). Physical violence against women in Santa Fe de Bogotá: prevalence and associated factors. Revista Panamericana de Salud Pública, 9, 78-83.

Kosek, R.B. (1996). The quest for a perfect spouse: Spousal ratings and marital satisfaction. Psychological Reports, 79, 731-735.

Lawrence, E., y Bradbury, T.N. (2001). Physical aggression and marital dysfunction: a 
longitudinal analysis. Journal of Family Psychology, 15, 135-154.

Lee, T.Y., Sun, G.H., y Chao SC. (2001). The effect of an infertility diagnosis on the distress, marital and sexual satisfaction between husbands and wives in Taiwan. Human Reproduction, 16, 1762-1767.

Lipman, E.L., MacMillan, H.L., y Boyle, M.H. (2001). Childhood abuse and psychiatric disorders among single and married mothers. American Journal of Psychiatry, 158, 73-77.

Locke, H.J., y Wallace, K.M. (1959). Shortterm marital adjustment and prediction test: Their reliability and validity. Journal of Marriage and Family Living, 21, 251255.

Milardo, R.M. (1998). Gender asymmetry in common couple violence. Personal Relationships, 5, 423-438.

Plichta, S.B., y Falik, M. (2001). Prevalence of violence ad its implications for women's health. Womens Health Issues, 11, 244258.

Ramos-Lira, L., Saltijeral-Méndez, M.T., Romero-Mendoza, M., Caballero-Gutiérrez, M.A., Martínez-Vélez, N.A. (2001). Frequency of different forms of sexual violence and its association with mental health problems. Salud Publica Mexicana, 43, 182-191.

Riehl, E.A., Frei, R., y Willi, J. (1994). People in divorce and their ambivalence: initial use of a newly developed couples inventory. Psychotherapy and Psychosomatic and Medical Psychology, 44, 37-45.

Riggs, D.S., Caulfield, M.B y Street, A.E. (2000). Risk for domestic violence: factors associated with perpetration and victimization. Journal of Clinical Psychology, 56, 1289-1316.

Robbins, E.M. (1999). Stress, coping resources, and couple satisfaction in depressed women. Dissertation Abstracts Internatio- nal: Section-B: The Sciences and Engineering, $59(8-B), 4482$.

Rosenberg, M. (1965): Society and the Adolescent Self Image. Princeton: Princeton University Press.

Scaturo, D.J., Hayes, T., Sagula, D., y Walter, T. (2000). The concept of codependency and its context within family systems theory. Family Therapy, 27, 63-70.

Shackelford, T.K., y Buss, D.M. (1997). Spousal esteem. Journal of Family Psychology, 11, 478-488.

Soler, H., Vinayak, P., y Quadagno, D. (2000). Biosocial aspects of domestic violence. Psychoneuroendocrinology, 25, 721-739.

Spielberger, C.D., Gorsuch, R.L., Lushene, R.E. (1970). Manual for the State/Trait Anxiety Inventory. Palo Alto, CA: Consulting Psychologist Press (Versión española, TEA, 1982)

Vincent, J.P., Weiss, R.L., y Birchler, G.R. (1975). A behavioral analysis of problem solving in distressed and nondistressed married and stranger dyads. Behavior Therapy, 6, 475-484.

Waltz, J., Babcock, J.C., Jacobson, N.S., y Gottman, J.M. (2000). Testing a typology of batterers. Journal of Consulting and Clinical Psychology, 68, 658-669.

Waring, E.M. (1994). The role of marital therapy in the treatment of depressed married women. Canadian Journal of Psychiatry, 39, 568-571.

Woods, S.J. (2000). Prevalence and patterns of posttraumatic stress disorder in and postabused women. Issues in Mental Health Nursery, 21, 309-324.

World Health Organization (WHO). (1996). Violence against women. Fact Sheet N 128. http://www who.int/inf-fs/en/fact 128.html.

World Health Organization (WHO). (1998). Gender and health a technical paper. http://www.who.int/frh-whd/GandH/ghreport/gendertech.htm. 
Alberto Espino y Begoña Olabarría (Coordinadores)(2003): La formación de los profesionales de la salud mental en España. Estado actual y perspectivas. Madrid: Asociación Española de Neuropsiquiatría.

En este texto se recogen las ponencias de la mesa que da título al libro, y que se presentaron en el último Congreso de la Asociación Española de Neuropsiquiatría (Oviedo, Junio 2003) sobre la formación de médicos, psicólogos y enfermeros especialistas en salud mental. Está estructurado en cuatro bloques que abordan distintos aspectos (situación (actual, propuestas y problemas) en materia de formación en Salud Mental en las tres disciplinas. El esquema que se sigue es la evolución profesional del formado: desde los estudios universitarios hasta la formación continuada de los profesionales, pasando por la formación específica.

Los dos primeros bloques contienen reflexiones acerca de la situación, contenidos, problemas y alternativas formativas en salud mental en los estudios universitarios (primera parte) y en la formación especializada (segunda parte). El tercer bloque se centra en algunas áreas de formación específica dentro de las propias especialidades, y en el cuarto bloque se discute sobre la formación continuada de los profesionales de la Salud Mental, formación no reglada y que está bajo la mirada de un gran número de instituciones que por así decirlo, «luchan» por su control.

La primera parte, "Los estudios universitarios sobre la Salud Mental: contenidos, problemas y alternativas", consta de tres capítulos referidos a la enseñanza de pregrado o universitaria de la psiquiatría en la licenciatura de medicina, de la psicología clínica en la licenciatura de psicología, y de la salud mental en la diplomatura de enfermería, y cuyos autores son, respectivamente, M. Gómez Beneyto, A. Belloch Fuster, y J. Fornés Vives. En cada uno de estos capítulos se hace un repaso a la evolución de las correspondientes disciplinas, desde su génesis hasta su implantación en las universidades españolas hasta llegar a su situación actual.

Los tres autores coinciden en denunciar el poco peso que las asignaturas relacionadas con la salud mental tienen respecto al total del pregrado. Menos de un $5 \%$ en el caso de la Medicina (dos asignaturas troncales: "Psicología médica" de 7 créditos y "Psiquiatría» de 9 créditos), menos de un $20 \%$ en el caso de la Psicología (tres asignaturas troncales de 9 créditos cada una: «Evaluación», "Personalidad» y «Psicopatología y tratamientos»), y menos del $10 \%$ en Enfermería (dos asignaturas de un mínimo de 6 créditos cada una: "Ciencias psicosociales aplicadas», y "Enfermería de Salud Mental y psiquiátrica"). Se denuncia asimismo la dispersión entre las distintas universidades en la enseñanza de la salud mental en psicología y enfermería (por ejemplo, la salud mental en enfermería, puede tener un peso desde 11 créditos a 18 créditos dependiendo de la universidad), lo que lleva a plantear la incógnita, según Belloch, de si en la situación actual cabe hablar de Licenciatura o más bien de Licenciaturas, al menos por lo que se refiere a Psicología, pues dependiendo de la Universidad donde se reciba la formación, esta puede ser totalmente diferente tanto en cuanto a peso relativo como, incluso, en orientaciones o contenidos. Los dos capítulos referidos a la enseñanza en Medicina y Psicología, coinciden también en denunciar la falta de interdisciplinariedad: no 
es usual que psiquiatras enseñen en las facultades de psicología, ni que psicólogos lo hagan en las de medicina. Y todos ellos señalan las deficiencias en la parte práctica de la formación. Por último, cada autor presenta sus propias sugerencias para una mejor enseñanza de la salud mental en los pregrados.

El segundo bloque, "La formación de especialidades en Salud Mental. Evolución, apuesta actual» está formado por cinco capítulos. Los tres primeros referidos a la especialidad de cada una de las disciplinas, el cuarto, a la necesidad de nuevos instrumentos para afrontar la interdisciplinariedad y la cooperación, y en el último, se hace un repaso a la evolución de la psiquiatría en el período de la transición a la democracia.

En los tres primeros capítulos, sus respectivos autores (A. Espino, B. Olabarría y f. Paredes), coinciden en la necesidad de incrementar en un año más la formación reglada de especialistas en salud mental en cada una de las disciplinas ( 5 años para la formación MIR, 4 años para la formación PIR, y 2 años para la formación del EIR). Sólo en el último caso, se ha alcanzado el incremento solicitado. Respecto a la formación de la especialidad psiquiátrica (capítulo primero), se hace un breve repaso a la evolución de la formación en psiquiatría desde que a finales del siglo XIX surge la Revista Frenopática en Barcelona, hasta el Real Decreto de 1984 que supone la institucionalización definitiva del sistema MIR. Se describen los hitos más importantes de la institucionalización académica (dentro de los estudios universitarios) y de la especialidad (dentro del Sistema Nacional de Salud). Así mismo, se comentan los factores que afectan a la calidad formativa, y se describe la práctica psiquiátrica actual, perfilando el rol del psiquiatra y señalando los problemas para establecer límites con otras disciplinas. Por último se denuncia la necesidad de una formación continuada, y la importancia de que nunca olvidemos preguntarnos qué es lo que se aprende con la formación MIR.

El capítulo segundo, es una revisión del proceso de institucionalización de la especialidad de psicología clínica, arrancando de 1969 con la creación de la titulación universitaria de Psicología, y finalizando con la Orden Ministerial del 2002 que supone el cierre de la fase de institucionalización oficial de la psicología clínica. Olabarría describe el marco normativo actual, la configuración de la formación, evaluación y seguimiento en psicología clínica, proponiendo cambios y mejoras, y analiza las implicaciones del Real Decreto de 1984 y de la Orden Ministerial de 1995 para la Psicología Clínica. Denuncia la carencia de un sistema reglado y oficialmente establecido para la formación continuada, así como los costes de la misma y el peso de intereses como los de la industria farmacológica. También reflexiona sobre el contexto de la formación de especialistas sanitarios y la formación en psicología clínica, y sobre la necesidad de integrar esta especialidad en nuevos ámbitos de la red sanitaria, denunciando la necesidad de considerar y aplicar el modelo biopsicosocial.

La formación de los enfermeros especialistas (EIR), se aborda en el tercer capítulo. La especialidad se crea en 1987, y once años después surge la especialidad de salud mental (EIR). En este capítulo se describe el programa práctico y teórico de la especialidad, y se denuncia que en el momento de escribir el capítulo (2002), aún no se ha constituido la Comisión Nacional de Especialidades.

En el cuarto capítulo se señala que las características de la salud mental, que desde la óptica de la atención comunitaria (Informe de la OMS-2001) debe apostar por la misma, hacen necesaria la coordinación entre los profesionales de salud 
mental, y el resto de disciplinas del sistema sanitario, y entre los de otros sistemas como el educativo y el de servicios sociales. Ya no se concibe un "servicio fuerte de salud mental» que de respuesta a todas las necesidades de los pacientes. Se apuesta por la atención comunitaria y por la superación de una visión reduccionista que se quede en los síntomas, el déficit y en los efectos de la enfermedad.

En el último capítulo, Espino relaciona la formación psiquiátrica con la transición a la democracia, incluyéndose una cronología de la transición política, y de las normativas de la formación postgraduada durante este período. Se describen los conflictos y desencuentros entre médicos internos y residentes y la administración, desde la regulación normativa de 1955 hasta el Real Decreto de Especialidades de 1978. Y se revisa la formación de los psiquiatras durante el proceso de regulación institucional descrito.

El tercer bloque, «Áreas de formación específica, instrumentos y técnicas de intervención en salud mental», está compuesto por siete capítulos, la mayoría de los cuales profundizan en Áreas de Especial Interés de la formación especializada sobre las que existen propuestas en marcha. Otros se centran en medios técnicos necesarios para los especialistas en salud mental.

En el primer capítulo, los autores (M. López, L. Lara y M. Laviana) exponen su propia visión sobre la Atención comunitaria en salud mental, que entienden como un enfoque (paradigma tecnológico) teórico, organizativo y funcional, alternativo a otros modelos como los basados en Hospitales Psiquiátricos. Definen los conceptos de rehabilitación y los programas de apoyo social, relacionándolos con el enfoque de la atención comunitaria en salud mental. Por último, se centran en la formación (objetivos y metodología) de profesionales de la atención comunitaria, y específicamente del personal de los equipos o centros comunitarios de salud mental (incluye enfermería diplomada, y auxiliar, trabajadores sociales, psicólogos clínicos, psiquiatras, terapeutas ocupacionales, y monitores).

El capítulo segundo (C. Escudero, E. Serrano) aborda la formación de especialistas de salud mental de niños y adolescentes, formación que se considera muy insuficiente en los programas MIR (4 meses) y PIR (6 meses). Los autores revisan la situación de la especialización en Europa, y los planteamientos de la Unión Europea de Médicos Especialistas y de la Federación Europea de Asociaciones de Psicólogos. Se comentan las características de las Áreas de Capacitación Específica de psiquiatría y psicología infantil que las respectivas comisiones nacionales de psiquiatría y psicología clínica están elaborando en España. Por último se aborda la formación continuada en esta área.

El tercer capítulo (A. Moriñigo) analiza la situación de la especialización en geriatría dentro del MIR de psiquiatría. En España se traduce en una rotación opcional de cuatro meses, mientras que en otros países es obligatoria, aunque también breve. Se propone la creación de una super-especialización o un incremento del currículum sobre psiquiatría geriátrica, y algunos de los objetivos que la misma debería alcanzar.

En el siguiente capítulo (A. Fernández-Liria, B. Rodríguez)) se abordan los aspectos esenciales para la formación en Psicoterapia, centrándose en la propuesta del Área de Capacitación Específica en Psicoterapia en la que están trabajando las Comisiones Nacionales de psiquiatría y psicología clínica. Ésta parte de una definición restrictiva de la psicoterapia como una intervención orientada al tratamiento de los trastornos mentales, y ofrece una perspectiva integradora de las aportaciones de las diferentes escuelas. 
El capítulo quinto (N. Caparrós), propone desde una perspectiva psicoanalítica, concretamente desde el modelo analítico-vincular, un plan de formación teórico (formación psicoanalítica centrada en el sujeto, y seminario sobre grupos (de duración mínima de tres años) y técnico (análisis personal y trabajo en grupo desempeñando escenas y situaciones "como si») en psicoterapia de grupo.

El siguiente capítulo, (F. Mansilla), se centra en la formación en drogodependencias y abuso de sustancias. Se denuncia la insuficiencia de la misma en el pregrado (no hay asignaturas regladas sobre drogodependencias) y en el postgrado (programa MIR de psiquiatría: formación mínima de 8 meses, y en el programa PIR no se especifica el tiempo). Así mismo, se hace una breve revisión de la formación no reglada, y de la evolución de la misma desde que en los años 70 , con el consumo de heroína, las drogodependencias se convierten en un problema de importancia creciente, y con características diferentes a las del consumo del alcohol. Por último, se proponen una serie de criterios, objetivos, contenidos y metodología a seguir en la formación en esta disciplina, y se sugiere la necesidad de disponer de un sistema de formación reglada y continuada dentro del sistema de salud.

En el último capítulo de esta tercera parte (J. Otero), se aborda la formación en psicofarmacología de los profesionales de la salud mental. Tras una breve introducción histórica sobre la psicofarmacología y la revolución que ha supuesto en la psiquiatría, se denuncia la poca importancia que se le concede en la licenciatura de medicina, y la creciente importancia que se le da en psicología, así como la escasa formación en el postgrado, la ausencia de unidades de psicofarmacología en los hospitales y la lucha por el poder de las empresas farmacológicas. Por último, se propone un guión que debería seguir cualquier curso de formación en psicofarmacología.

El cuarto bloque es el más polémico, y bajo el título "Formación continuada, ejercicio profesional y perspectivas complementarias», incluye seis capítulos que abarcan diferentes dimensiones de la formación no reglada en salud mental.

En el primer capítulo (V. Pereira, M.V. Rodríguez) se describe cómo influye y qué repercusiones tiene la industria farmacológica sobre la formación continuada de los médicos españoles y sobre la dirección de la investigación, así como las consecuencias de esta realidad sobre el quehacer médico y el creciente gasto farmacológico. Se sintetizan algunas directrices y códigos propuestos por diferentes instituciones y comités de bioética para regular la relación de los médicos con la industria farmacológica, y se concluye señalando la necesidad de que las administraciones públicas intervengan sobre esta problemática, asumiendo la formación continuada, y poniendo en funcionamiento el «Sistema Español de Acreditación de la formación Médica Continuada».

En el segundo capítulo (J. Leal) se aborda la supervisión de equipos, tanto clínica como institucional, en el ámbito de la formación continuada en salud mental. Ésta crea un espacio grupal-institucional en el que un equipo multidisciplinar de profesionales, expone su trabajo (que se pretende sea interdisciplinar) a un tercero ajeno a la cotidianeidad del equipo, el supervisor, con la intención de recibir aportes que tengan efectos sobre el aprendizaje y la mejora de la práctica profesional. Se describen diferentes tipos de demanda, y los procesos que subyacen a cada tipo de supervisión, así como las implicaciones éticas y características que debe tener su figura.

El capítulo tercero (E. Sentís y J. Ramos) describe al profesional de la salud mental no sólo como un técnico o 
experto, sino como un regulador del propio sistema que necesita adquirir conocimientos de gestión clínica en su formación de especialista. Tras describirlos, se insiste en la importancia y beneficios de que sea el propio profesional de la salud mental, el que opte por la gestión de servicios en su campo, y se indican algunos de los criterios que estos profesionales deben cumplir.

En el cuarto capítulo (P. Pérez) se expone la importancia de cuidar los aspectos emocionales de la relación médicopaciente, así como la necesidad de formar subequipos interdisciplinares. Se introduce un modo de pensar psicosomático, se presentan distintos grupos de trabajo, y se insiste en la importancia de los mismos en la protección del desgaste que sufre el equipo, y en su función de técnicas para la formación psicológica del profesional sanitario.

El quinto capítulo (A. Ávila) describe la evolución, durante la última década, de los esfuerzos y dificultades desarrolladas para llegar a un consenso sobre la formación y reconocimiento de la psicoterapia en el marco europeo. Se describen los aspectos más importantes del mejor consenso actual (2002), el del documento de Munich del año 2001 ( $« \mathrm{La}$ profesión de psicoterapia en Europa»), y analiza los logros, consecuencias, objetivos, y próximos retos a superar.

El último capítulo de esta parte y del libro (L. Hernangómez y V. Suárez), presenta un resumen de los datos extraídos de una encuesta rellenada por 45 PIR y psicólogos clínicos vía PIR, sobre el desarrollo de la formación recibida durante su residencia. La valoración se realiza sobre cuatro grandes áreas: rotaciones, forma- ción teórica y práctica, y valoración general. Los resultados indican una valoración global positiva, no obstante lo cual se sugieren propuestas de mejora.

En definitiva, este texto recoge los capítulos más importantes sobre la historia de la Salud Mental en España, relatados por muchos de sus protagonistas más representativos. Es un retrato y análisis de la situación actual, de lo existente, de sus problemas, y posibles soluciones, y es también un libro que recoge nuevas posiciones y actitudes, lleno de propuestas de futuro, que llama a la reflexión, marca horizontes y nuevas vías de desarrollo posibles y deseables. Es una historia reciente y cercana para gran parte de los profesionales de la salud mental española, y por ello es un libro que a unos les resultará interesante porque reúne la evolución vivida en la salud mental española en las últimas décadas, y por sus propuestas, y a otros, los nuevos profesionales de la salud mental, les ayudará a entender mejor el estado de la cuestión, a comprender los cambios que se están dando en el marco de las especialidades, así como porque dan a conocer vías de mejora en la atención en salud mental. En todos los casos, considero que se trata de un texto imprescindible para comprender la situación en la que nos encontramos actualmente en torno a la salud mental y es representativo de la multidisciplinariedad que tantas veces se reclama, pero que en tan pocas ocasiones se logra.

Gemma García Soriano Becaria FPU

Facultad de Psicología, Universidad de Valencia 